\title{
ON THE GROWTH OF CERTAIN MEROMORPHIC SOLUTIONS OF ARBITRARY SECOND ORDER ALGEBRAIC DIFFERENTIAL EQUATIONS ${ }^{1}$
}

\author{
STEVEN BANK
}

\begin{abstract}
In this note, we present two results concerning meromorphic functions on the whole finite plane, which are solutions of algebraic differential equations (i.e., equations of the form $\Omega\left(z, y, d y / d z, \cdots, d^{n} y / d z^{n}\right)=0$, where $\Omega$ is a polynomial in $\left.z, y, d y / d z, \cdots, d^{n} y / d z^{n}\right)$.
\end{abstract}

1. Introduction. Our main theorem (Theorem 1 below) concerns the growth of certain meromorphic solutions of second order equations. In [8, pp. 221-223], Valiron (and also Wittich [10, pp. 70-71]) treated a very special class of second and higher order algebraic differential equations, and it was shown that all entire solutions of equations in this special class were of finite order of growth. (See also Nikolaus [6, p. 625].) Of course, arbitrary second order algebraic differential equations may possess entire solutions of infinite order and our Theorem 1 deals with certain of these solutions. More specifically, Theorem 1 treats any meromorphic solution $y_{0}(z)$ with the property that for two distinct values of $\lambda$ (finite or infinity), the sequence of moduli of the nonzero roots of the equation $y_{0}(z)=\lambda$ has a finite exponent of convergence [4, p. 188]. (Of course, by a theorem of Nevanlinna [5, p. 72], there can be at most two such values of $\lambda$ for a meromorphic function of infinite order.) We show that the Nevanlinna characteristic $T\left(r, y_{0}\right)$ of such a solution must satisfy an inequality of the form, $T\left(r, y_{0}\right) \leqq \exp \left(r^{A}\right)$ for some positive constant $A$ and all $r$ greater than some number $r_{0}$.

The proof of Theorem 1 involves first proving a preliminary result (Theorem 2) which sets forth a sufficient condition for a meromorphic solution of an $n$th order algebraic differential equation $\Omega=0$, to necessarily be a solution of each equation $\Omega_{q}=0$, where $\Omega_{q}$ is the homogeneous part of $\Omega$ of degree $q$ in the indeterminates $y, y^{\prime}, \ldots$, $y^{(n)}$. The proof of Theorem 2 involves standard Nevanlinna theory

Received by the editors December 3, 1969.

AMS Subject Classifications. Primary 3406, 3442, 3061.

Key Words and Phrases. Algebraic differential equations, differential polynomials, growth of meromorphic functions, meromorphic solutions, Nevanlinna characteristic, exponent of convergence.

${ }^{1}$ This research was supported in part by the National Science Foundation GP11798. 
and a result of Clunie [2, Lemma 1]. Theorem 1 is then proved using Theorem 2 and a result from [1] concerning first order equations, which states that a meromorphic solution of a first order algebraic differential equation must be of finite order if the sequence of moduli of its poles has a finite exponent of convergence. (This result from [1] generalizes the well-known result of Valiron [9] that an entire solution of a first order equation must be of finite order.)

2. We now state our main result.

THEOREM 1. Let $y_{0}(z)$ be a meromorphic solution of a second order algebraic differential equation such that for some rational function $R(z)$, there are two distinct values of $\lambda$ (finite or infinite) for which the sequence of moduli of the roots of the equation $y_{0}(z)-R(z)=\lambda$ has a finite exponent of convergence. Then there exist real numbers $A>0$ and $r_{0} \geqq 0$ such that $T\left(r, y_{0}\right) \leqq \exp \left(r^{A}\right)$ for all $r \geqq r_{0}$.

The proof will be given in $\$ 5$.

3. Notation. For a meromorphic function $f(z)$, we will use the standard notation for the Nevanlinna functions $m(r, f), N(r, f), T(r, f)$ and $\bar{N}(r, f)$ introduced in [5, pp. 6, 12, 70]. Following Clunie [2], we use the abbreviation n.e. (nearly everywhere) to mean "everywhere in $[0,+\infty)$ except in a set of finite measure." Finally, if $\Omega\left(z, y, y^{\prime}, \cdots, y^{(n)}\right)$ is a polynomial in $z, y, \cdots, y^{(n)}$, then for each nonnegative integer $q$, we denote by $\Omega_{q}$ the homogeneous part of $\Omega$ of degree $q$ in the indeterminates $y, y^{\prime}, \cdots, y^{(n)}$.

4. We now prove

Theorem 2. Let $\Omega\left(z, y, y^{\prime}, \cdots, y^{(n)}\right)$ be a polynomial in $z, y$, $y^{\prime}, \cdots, y^{(n)}$, and let $y_{0}(z)$ be a nonconstant meromorphic solution of the differential equation $\Omega=0$. Then if for some nonnegative integer $q$, the function $y_{0}(z)$ is not a solution of the equation $\Omega_{q}=0$, then we must have n.e. as $r \rightarrow+\infty$,

$$
T\left(r, y_{0}\right) \leqq 2 p N\left(r, 1 / y_{0}\right)+(2 \gamma p+1) N\left(r, y_{0}\right)+2 \gamma \sigma \bar{N}\left(r, y_{0}\right)
$$

$$
+O\left(\log r+\log T\left(r, y_{0}\right)\right)
$$

where $p$ is the maximum nonnegative integer with the property that $y_{0}(z)$ is not a solution of $\Omega_{p}=0$, and where $\sigma$ and $\gamma$ are respectively, the maximum weight and the number of nonzero terms in $\Omega_{p}$ (when $\Omega_{p}$ is viewed as a polynomial in the indeterminates $\left.y, y^{\prime}, \cdots, y^{(n)}\right)$.

With $p$ as defined above, we have for all $z$ that, 
(2) $\Omega_{p}\left(z, y_{0}(z), \cdots, y_{0}^{(n)}(z)\right)=-\sum_{t \leqq p-1} \Omega_{t}\left(z, y_{0}(z), \cdots, y_{0}^{(n)}(z)\right)$.

We denote the left side of (2) by $E(z)$ and the right side by $F(z)$. By assumption $E(z) \not \equiv 0$ and so $F(z) \not \equiv 0$. Now $F(z)$ is a differential polynomial of degree at most $p-1$ in $y_{0}, y_{0}^{\prime}, \cdots, y_{0}^{(n)}$, and has polynomial coefficients. Hence if we set $G=F / y_{0}^{p-1}$, then by [2, Lemma 1], we have n.e. as $r \rightarrow+\infty$, that

$$
\frac{1}{2 \pi} \int_{\left|y_{0}\right|>1} \log ^{+}\left|G\left(r e^{i \theta}\right)\right| d \theta=O\left(\log r+\log T\left(r, y_{0}\right)\right) .
$$

Now (2) may be written,

$$
\left(y_{0}^{-p} E\right) y_{0}=G,
$$

and hence when $\left|y_{0}(z)\right| \leqq 1$, we have $|G(z)| \leqq\left|\left(y_{0}(z)\right)^{-p} E(z)\right|$. Thus clearly, for each $r$,

$$
\frac{1}{2 \pi} \int_{\left|y_{0}\right| \leqq 1} \log ^{+}\left|G\left(r e^{i \theta}\right)\right| d \theta \leqq m\left(r, y_{0}^{-p} E\right) .
$$

Thus by (3) and (5), we have n.e. as $r \rightarrow+\infty$ that

$$
m(r, G) \leqq m\left(r, y_{0}^{-p} E\right)+O\left(\log r+\log T\left(r, y_{0}\right)\right) .
$$

Now $E(z)$ is a sum of terms of the form,

$$
U=f y_{0}^{j_{0}}\left(y_{0}^{\prime}\right)^{j_{1}} \cdots\left(y_{0}^{(n)}\right)^{j_{n}},
$$

where $j_{0}+j_{1}+\cdots+j_{n}=p$ and $f$ is a polynomial. Thus $y_{0}^{-p} E$ is a sum of terms of the form $V=f\left(y_{0}^{\prime} / y_{0}\right)^{j_{1}} \cdots\left(y_{0}^{(n)} / y_{0}\right)^{j_{n}}$. Thus (using [5, p. $14])$, it follows that

$$
m(r, V)=O\left(\log r+\sum_{k=1}^{n} m\left(r, y_{0}^{(k)} / y_{0}\right)\right) \quad \text { as } r \rightarrow+\infty .
$$

But

$$
m\left(r, y_{0}^{(k)} / y_{0}\right) \leqq \sum_{j=0}^{k-1} m\left(r, y_{0}^{(j+1)} / y_{0}^{(j)}\right)
$$

and by $[5$, p. 63$]$,

$$
m\left(r, y_{0}^{(j+1)} / y_{0}^{(j)}\right)=O\left(\log r+\log T\left(r, y_{0}^{(j)}\right)\right) \text { n.e. } \quad \text { as } r \rightarrow+\infty .
$$

Since also $T\left(r, y_{0}^{(j)}\right)=O\left(T\left(r, y_{0}\right)\right)$ n.e. as $r \rightarrow+\infty$ (by $[3$, p. 55]), it now 
easily follows from the above estimates that

$$
m\left(r, y_{0}^{-p} E\right)=O\left(\log r+\log T\left(r, y_{0}\right)\right) \text { n.e. } \quad \text { as } r \rightarrow+\infty .
$$

Now from (4) and [5, p. 14], $N(r, G) \leqq N\left(r, y_{0}^{-p} E\right)+N\left(r, y_{0}\right)$ and since $y_{0}=G /\left(y_{0}^{-p} E\right)$, we have

$$
T\left(r, y_{0}\right) \leqq T(r, G)+T\left(r, y_{0}^{-p} E\right)+O(1) \quad \text { as } r \rightarrow+\infty .
$$

It therefore follows using (6) and (8) that n.e. as $r \rightarrow+\infty$,

$$
T\left(r, y_{0}\right) \leqq 2 N\left(r, y_{0}^{-p} E\right)+N\left(r, y_{0}\right)+O\left(\log r+\log T\left(r, y_{0}\right)\right) .
$$

We now consider $N\left(r, y_{0}^{-p} E\right)$. We have,

$$
N\left(r, y_{0}^{-p} E\right) \leqq p N\left(r, 1 / y_{0}\right)+N(r, E) .
$$

Now $E$ is the sum of $\gamma$ terms of the form $U$ given in (7), and using [5, p. 14] we have as $r \rightarrow+\infty$,

$$
N(r, U) \leqq O(\log r)+j_{0} N\left(r, y_{0}\right)+\cdots+j_{n} N\left(r, y_{0}^{(n)}\right) .
$$

By [5, p. 104], $N\left(r, y_{0}^{(k+1))}\right)=N\left(r, y_{0}^{(k)}\right)+\bar{N}\left(r, y_{0}^{(k)}\right)$, and it is easily verified that $\bar{N}\left(r, y_{0}^{(k)}\right)=\bar{N}\left(r, y_{0}\right)$. It now follows by induction on $k$ that $N\left(r, y_{0}^{(k)}\right)=N\left(r, y_{0}\right)+k \bar{N}\left(r, y_{0}\right)$. Since $j_{0}+j_{1}+\cdots+j_{n}=p$, it therefore follows from (12) that

$$
N(r, U) \leqq O(\log r)+p N\left(r, y_{0}\right)+w(j) \bar{N}\left(r, y_{0}\right),
$$

where $w(j)$ is the weight $j_{1}+2 j_{2}+\cdots+n j_{n}$. Thus clearly, as $r \rightarrow+\infty$,

$$
N(r, E) \leqq O(\log r)+\gamma p N\left(r, y_{0}\right)+\gamma \sigma \bar{N}\left(r, y_{0}\right),
$$

where $\gamma$ and $\sigma$ are as in the statement of the theorem. The conclusion (1) of the theorem now follows immediately from (10), (11) and (14).

5. Proof of Theorem 1. We may assume that $y_{0}(z)$ is of infinite order since the result clearly holds if $y_{0}(z)$ is of finite order. Set $u_{0}(z)=y_{0}(z)-R(z)$. Then $u_{0}$ is clearly also of infinite order since $R(z)$ is rational. Let $\lambda_{1}$ and $\lambda_{2}$ be the two values of $\lambda$ referred to in the statement. If $\lambda_{1}$ and $\lambda_{2}$ are both finite, set $v_{0}=\left(u_{0}-\lambda_{2}\right) /\left(u_{0}-\lambda_{1}\right)$. If one, say $\lambda_{1}$, is infinite, set $v_{0}=u_{0}-\lambda_{2}$. If both $\lambda_{1}$ and $\lambda_{2}$ are finite, then $y_{0}=R+\left(\left(\lambda_{1} v_{0}-\lambda_{2}\right) /\left(v_{0}-1\right)\right)$. If we differentiate this relation twice and substitute the resulting expressions for $y_{0}, y_{0}^{\prime}$ and $y_{0}^{\prime \prime}$ into the differential equation satisfied by $y_{0}$, then after multiplication by a suitable polynomial and a suitable power of $v_{0}-1$, we see that $v_{0}$ is a solution of a second order algebraic differential equation. We may write this equation in the form, 


$$
\Lambda\left(z, v, v^{\prime}, v^{\prime \prime}\right)=\sum f_{i j k}(z) v^{i}\left(v^{\prime}\right)^{j}\left(v^{\prime \prime}\right)^{k}=0,
$$

where the $f_{i j k}$ are polynomials. (A similar proof shows that this conclusion also holds when $\lambda_{1}=\infty$ (i.e., when $\left.y_{0}=R+\lambda_{2}+v_{0}\right)$.)

Now from the construction of $v_{0}$ (and the assumptions about $\lambda_{1}$ and $\lambda_{2}$ ), it is clear that both the sequence of moduli of the zeros of $v_{0}$ and the sequence of moduli of the poles of $v_{0}$ have finite exponents of convergence. Hence by [4, p. 195], we can form the canonical product $\phi(z)$ whose sequence of zeros is the sequence of zeros of $v_{0}$, and we can form the canonical product $\psi(z)$ whose sequence of zeros is the sequence of poles of $v_{0}$. Then by [4, p. 195],

$$
\phi \text { and } \psi \text { are entire functions of finite order. }
$$

Thus there exists $a>0$ such that

$$
T(\boldsymbol{r}, \boldsymbol{\phi})=O\left(\boldsymbol{r}^{a}\right) \quad \text { and } \quad T(\boldsymbol{r}, \psi)=O\left(\boldsymbol{r}^{a}\right) \quad \text { as } r \rightarrow+\infty .
$$

By construction, $\psi v_{0} / \phi$ is an entire function which is nowhere zero. Thus there exists an entire function $g$ such that

$$
v_{0}=(\phi / \psi) e^{g} .
$$

We note also that since $u_{0}$ is of infinite order and since $T\left(r, u_{0}\right)$ $=T\left(r, v_{0}\right)+O(1)$ as $r \rightarrow+\infty$ (by $[5$, p. 14]), we have that

$$
v_{0} \text { is of infinite order. }
$$

Now by [5, p. 14], $T(r, 1 / \phi)=T(r, \phi)+O(1)$ as $r \rightarrow+\infty$, and hence by (17) and the definition of $\phi$, we obtain,

$$
N\left(r, 1 / v_{0}\right)=N(r, 1 / \phi)=O\left(r^{a}\right) \quad \text { as } r \rightarrow+\infty .
$$

Similarly, by (17) and the definition of $\psi$ we obtain,

$$
N\left(r, v_{0}\right)=N(r, 1 / \psi)=O\left(r^{a}\right) \quad \text { as } r \rightarrow+\infty .
$$

We now assert that $v_{0}$ does not satisfy the condition (1) of Theorem 2. To prove this, we assume the contrary. Then in view of (20), (21) and the fact that $\bar{N}\left(r, v_{0}\right) \leqq N\left(r, v_{0}\right)$ for $r \geqq 1$, we would obtain $T\left(r, v_{0}\right) \leqq K\left(r^{a}+\log T\left(r, v_{0}\right)\right)$ n.e. for some constant $K>0$. But since $T\left(r, v_{0}\right) \rightarrow+\infty$ as $r \rightarrow+\infty$ (by (19)), we have $K\left(\log T\left(r, v_{0}\right)\right.$ ) $\leqq(1 / 2) T\left(r, v_{0}\right)$ for all sufficiently large $r$. Thus the inequality, $T\left(r, v_{0}\right) \leqq 2 K r^{a}$ would hold for all $r$ not belonging to some set $B$ of finite measure. Let $\alpha$ be a number greater than the measure of $B$, and let $r$ be any number greater than $\alpha$. Then clearly $[r, r+\alpha]$ cannot be contained in $B$, so there exists $s \in[r, r+\alpha]$ such that $s \notin B$. Thus $T\left(s, v_{0}\right) \leqq 2 K s^{a}$. Since $T$ is increasing and since $s \leqq r+\alpha \leqq 2 r$, we would 
thus obtain $T\left(r, v_{0}\right) \leqq 2^{a+1} K r^{a}$ for all $r>\alpha$, which of course contradicts (19). This contradiction proves that $v_{0}$ does not satisfy (1) of Theorem 2. Since $v_{0}$ is a solution of the equation (15), we have by Theorem 2 , that for each $q \geqq 0, v_{0}$ is a solution of the equation $\Lambda_{q}\left(z, v, v^{\prime}, v^{\prime \prime}\right)=0$. Setting $p=\max \left\{i+j+k: f_{i j k} \not \equiv 0\right\}$ (where the $f_{i j k}$ are as in (15)), then clearly $p>0$ and $\Lambda_{p}\left(z, v_{0}, v_{0}^{\prime}, v_{0}^{\prime \prime}\right) \equiv 0$. Thus

$$
\sum_{i+j+k=p} f_{i j k}(z) v_{0}^{i}\left(v_{0}^{\prime}\right)^{j}\left(v_{0}^{\prime \prime}\right)^{k} \equiv 0
$$

Now set $w_{0}=v_{0}^{\prime} / v_{0}$. Then dividing the relation (22) by $v_{0}^{p}$ (and noting that $v_{0}^{\prime \prime} / v_{0}=w_{0}^{\prime}+w_{0}^{2}$ ), we see that $w_{0}$ is a meromorphic solution of the first order algebraic differential equation,

$$
\sum_{i+j+k=p} f_{i j k}(z) w^{j}\left(w^{\prime}+w^{2}\right)^{k}=0 .
$$

Now any pole of $w_{0}$ is simple and is either a zero or a pole of $v_{0}$. Since both the sequence of moduli of the zeros of $v_{0}$ and the sequence of moduli of the poles of $v_{0}$ have finite exponents of convergence, it is clear that the sequence of moduli of the poles of $w_{0}$ has a finite exponent of convergence. It is proved in $[1, \S 4]$, that a meromorphic function with this property, which is a solution of a first order algebraic differential equation, must be of finite order. Thus $w_{0}$ is of finite order.

Now, from (18) we have,

$$
g^{\prime}=w_{0}-\left(\phi^{\prime} / \phi\right)+\left(\psi^{\prime} / \psi\right) .
$$

In view of (16), we have by [5, p. 63] that $m\left(r, \phi^{\prime} / \phi\right)=O(\log r)$ and $m\left(r, \psi^{\prime} / \psi\right)=O(\log r)$ as $r \rightarrow+\infty$. Since $\phi$ is entire, $N\left(r, \phi^{\prime}\right)=0$ so $N\left(r, \phi^{\prime} / \phi\right) \leqq N(r, 1 / \phi)$. Thus by $(20), N\left(r, \phi^{\prime} / \phi\right)=O\left(r^{a}\right)$ as $r \rightarrow+\infty$. Similarly, $N\left(r, \psi^{\prime} / \psi\right)=O\left(r^{a}\right)$, and hence we obtain,

$$
T\left(r, \phi^{\prime} / \phi\right)=O\left(r^{a}\right) \text { and } T\left(r, \psi^{\prime} / \psi\right)=O\left(r^{a}\right) \quad \text { as } r \rightarrow+\infty \text {. }
$$

In view of (24), (25) and (26) and [5, p. 14], it follows that $g^{\prime}$ is of finite order. But by $[7$, p. 323], the entire function $g$ and its derivative are of the same order. Thus $g$ is also of finite order, so there exist $b>0$ and $r_{1}>0$ such that

$$
M(r, g) \leqq \exp \left(r^{b}\right) \quad \text { for all } r \geqq r_{1},
$$

where $M(r, g)$ is the maximum modulus of $g(z)$ on $|z|=r$. But since $M\left(r, e^{o}\right) \leqq \exp (M(r, g))$, we thus have $\log ^{+} M\left(r, e^{o}\right) \leqq \exp \left(r^{b}\right)$, and hence, 


$$
T\left(r, e^{\theta}\right) \leqq \exp \left(r^{b}\right) \quad \text { for all } r \geqq r_{1} .
$$

Thus from (17), (18) and [5, p. 14], it follows easily that for some $r_{2}>0$ we have,

$$
T\left(r, v_{0}\right) \leqq 2 \exp \left(r^{b}\right) \quad \text { for all } r \geqq r_{2} .
$$

But by [5, p. 14], $T\left(r, u_{0}\right)=T\left(r, v_{0}\right)+O(1)$, and since $R(z)$ is rational, $T\left(r, y_{0}\right) \leqq T\left(r, u_{0}\right)+O(\log r)$ as $r \rightarrow+\infty$. Thus from (29), it clearly follows that for any $\epsilon>0$, there exists $r_{0}=r_{0}(\epsilon)$ such that $T\left(r, y_{0}\right)$ $\leqq \exp \left(r^{b+\epsilon}\right)$ for all $r \geqq r_{0}$. This concludes the proof of Theorem 1 .

\section{REFERENCES}

1. S. Bank, On meromorphic solutions of algebraic differential equations, Comment. Math. Helv. 44 (1969), 401-409.

2. J. Clunie, On integral and meromorphic functions, J. London Math. Soc. 37 (1962), 17-27. MR $26 \# 1456$.

3. W. K. Hayman, Meromorphic functions, Oxford Math. Monographs, Clarendon Press, Oxford, 1964. MR 29 \#1337.

4. E. Hille, Analytic function theory. Vol. II, Introductions to Higher Math., Ginn, Boston, Mass., 1962. MR 34 \#1490.

5. R. Nevanlinna, Le théorème de Picard-Borel et la théorie des functions méromorphes, Gauthier-Villars, Paris, 1929.

6. J. Nikolaus, Über ganze Lösungen linearer Differentialgleichungen, Arch. Math. (Basel) 18 (1967), 618-626. MR $37 \# 4307$.

7. S. Saks and A. Zygmund, Analytic functions, Monografie Mat., Tom 10, PWN, Warsaw, 1938; English transl., Tom 28, 1952. MR 14, 1073.

8. G. Valiron, Fonctions analytiques, Presses Universitaires de France, Paris, 1954. MR 15, 861.

9. - Sur les fonctions entières vérifiant une classe d'équations différentielles, Bull. Soc. Math. France 51 (1923), 33-45.

10. H. Wittich, Neuere Untersuchungen über eindeutige analytische Funktionen, Ergebnisse der Mathematik und ihrer Grenzgebiete, Heft 8, Springer-Verlag, Berlin and New York, 1955. MR 17, 1067.

UNIVERSITY OF IlLiNoIS, URBANA, ILlinOIS 61801 